\title{
Análisis psicométrico del Inventario de Autoeficacia para las Inteligencias Múltiples-Revisado (IAMI-R) en estudiantes peruanos de nivel secundario
}

\section{Psychometric Analysis of the Self-Efficacy Inventory for Multiple Intelligences-Revised (SIMI-R) in Peruvian High School Students}

\author{
Jaime Aliaga Tovar* (D) Universidad San Ignacio de Loyola, Lima, Perú \\ ORCID: https://orcid.org/0000-0002-4091-3205 \\ Carlos Ponce Díaz Universidad San Ignacio de Loyola, Lima, Perú \\ Universidad Inca Garcilaso de la Vega, Lima, Perú. \\ ORCID: https://orcid.org/0000-0002-0101-2244
}

Edwin Salas-Blas* (D) Universidad de San Martín de Porres, Lima, Perú. ORCID: https://orcid.org/0000-0002-0625-0313

Recibido 08-08-18 Revisado 20-08-18 Aprobado 11-10-18 En línea 16-10-18

*Correspondencia

Email: e.salasb@hotmail.com
Citar como:

Aliaga, J., Ponce, C., \& Salas-Blas, E. (2018). Análisis psicométrico del Inventario de

Autoeficacia para las Inteligencias MúltiplesRevisado (IAMI-R) en estudiantes peruanos de nivel secundario. Propósitos y Representaciones, 6(2), 63-94. Doi: http:// dx.doi.org/10.20511/pyr2018.v6n2.249

(C) Universidad San Ignacio de Loyola, Vicerrectorado de Investigación, 2018

(cc) BY-NC-ND Este artículo está distribuido bajo licencia CC BY-NC-ND 4.0 Internacional (http://creativecommons.org/licenses/by-nc-nd/4.0/). 


\section{Resumen}

El presente estudio instrumental (Ato, López y Benavente, 2013) o de tipo psicométrico (Alarcón, 2008) planteó como objetivo estimar la validez y fiabilidad del Inventario de Autoeficacia para las Inteligencias MúltiplesRevisado (IAMI-R, Pérez y Cupani, 2008) en estudiantes peruanos de quinto de secundaria. Se trabajó con una muestra de 1304 adolescentes, edad promedio 16.81, dividida en dos grupos de 652. La confiabilidad se calculó con el Alfa ordinal (Contreras \& Novoa-Muñoz, 2018) y con el coeficiente Omega de McDonald, obteniéndose valores apropiados. El Análisis Factorial Exploratorio (AFE) confirma los ocho factores de la versión original que explican el $70 \%$ de la variabilidad de las puntuaciones. Con el análisis factorial confirmatorio (AFC) se obtuvieron índices de ajuste que confirman esta estructura factorial: $\mathrm{SS} \chi 2=1857.73, \mathrm{gl}=1047, \mathrm{SS} \chi 2 / \mathrm{gl}=1.77$, $\mathrm{RMSEA}=.035, \mathrm{SRMR}=.049, \mathrm{CFI}=.915 \mathrm{y} \mathrm{TLI}=.908$. Los resultados confirman que el IAMI-R es adecuado para evaluar autoeficacia asociada a inteligencias múltiples en estudiantes peruanos que culminan la secundaria.

Palabras Clave: Autoeficacia para inteligencias múltiples, análisis psicométrico, estudiantes peruanos. 


\section{Summary}

The objective of this instrumental study (Ato, López \& Benavente, 2013) or psychometric-type (Alarcón, 2008) was to estimate the validity and reliability of the Self-Efficacy Inventory for Multiple Intelligences-Revised (IAMI-R, Pérez \& Cupani, 2008) in Peruvian students of fifth year of high school. We worked with a sample of 1304 adolescents, with an average age of 16.81, divided into two groups of 652 . Reliability was calculated with the Ordinal Alpha (Contreras \& Novoa-Muñoz, 2018) and with the McDonald's Omega coefficient, obtaining appropriate values. The Exploratory Factor Analysis (AFE) confirms the eight factors of the original version that explain $70 \%$ of the variability of the scores. With the confirmatory factorial analysis (CFA), adjustment indices were obtained confirming this factorial structure: $\mathrm{SS} \chi 2=1857.73, \mathrm{gl}=1047, \mathrm{SS} \chi 2 / \mathrm{gl}=1.77$, RMSEA $=.035, \mathrm{SRMR}=.049$, $\mathrm{CFI}=.915$, and $\mathrm{TLI}=.908$. The results confirm that the IAMI-R is adequate to evaluate self-efficacy associated with multiple intelligences in Peruvian students who complete high school.

Keywords: Self-efficacy for multiple intelligences, psychometric analysis, Peruvian students. 


\section{Introducción}

El contexto social en el que interacciona la persona tiene un rol muy importante en lo que éste aprende, Bandura (1977a), sobre la base de ciertos aspectos del condicionamiento clásico y operante, pero discrepando con estos modelos tradicionales introduce la noción de procesos cognoscitivos que median entre la estimulación y la respuesta y que tienen naturaleza interna y privada; a la vez, afirma que gran parte de lo que aprenden las personas se realiza por medio de la observación, que los observadores no desarrollan las conductas aprendidas en el mismo momento en que aprenden y que el reforzamiento no era indispensable para que se produzca el aprendizaje (Schunk, 2012). Conceptos claves de este modelo teórico son la imitación, la identificación, el modelado y el aprendizaje vicario, con los que se explica la adquisición de diferentes tipos de comportamientos, habilidades, creencias y estrategias. Más adelante identifica otros conceptos que fueron claves de su modelo teórico, como la autosuficiencia, autoeficacia, autoconfianza y la autoinstrucción como factores determinante en la regulación y en el éxito de la conducta dirigida a resultados (Bandura, 1977b, 1986, 1995, 1997) y que se constituyen en elementos medulares de su teoría; a partir de estos empiezan a participar otros conceptos como la auto-organización, autorregulación, autoreflexión, determinantes personales, ambientales, comportamentales y el determinismo recíproco, que perfilan la teoría hacia una definitiva visión cognitiva del aprendizaje.

La autoeficacia es uno de los conceptos centrales de la teoría de Bandura (1986), sostiene que son "los juicios de las personas sobre sus capacidades para organizar y ejecutar cursos de acción requeridos para alcanzar determinados niveles de desempeño" (p. 386). Para él los niveles de motivación, los estados afectivos y las acciones de las personas se fundamentan en las creencias que ellos tienen sobre lo que pueden realizar, más que en lo que realmente pueden ser capaces de realizar teniendo en cuenta criterios objetivos; éstas creencias acerca de lo que cada persona tiene acerca de lo que es capaz de hacer viene a constituir la base de la autoeficacia (Alegre, 2014; Bandura, 
1977b, 1995, 1997). De modo tal, que se puede sostener una especie de regla, si una persona posee un juicio pobre de sus propias capacidades puede esto ocasionar que ella rehúya las situaciones-problema, desarrolle comportamientos de evitación, o, afrontar las dificultades y adversidades que se presentan como obstáculos para cumplir con sus objetivos de vida y entrar en desánimo. Contrariamente si la persona posee creencias positivas de que puede afrontar el problema y resolverlo, esa condición facilitará un afronte directo y más posibilidades de solucionar el problema (Alegre, 2014; Lazarus \& Folkman, 1986; Piergiovanni \& Depaula, 2018; entre otros).

Sin embargo, no todo es creencia y alta expectativa por parte del individuo, la autoeficacia resulta una creencia central que tiene capacidad para condicionar y activar varios procesos cognitivos, motivacionales, afectivos y selectivos (Covarrubias \& Mendoza, 2013). Pero, la ejecución satisfactoria de una actividad en una situación o tarea específica, requiere de un equilibrio entre la autoeficacia y la posesión de las capacidades y habilidades para su ejecución (Gálvez, Chía \& Valdez, 2005; Naranjo, 2009), además del juicio sobre el resultado más probable de una conducta determinada (Olaz, 2001).

Asimismo, Bandura (1997) explica que las personas pueden controlar muchos acontecimientos considerados importantes por él a través de la autorregulación, que es aplicable tanto a pensamientos como a acciones. Autorregular supone establecer metas y adelantarse mediante el cálculo a los resultados que tendrán sus acciones en el futuro. Bandura (1986) sostenía "Las personas no actúan sólo para ajustarse a las preferencias de los demás; gran parte de su conducta es motivada y regulada por estándares internos y respuestas de autoevaluación de sus propias acciones" (p. 20).

Por otro lado, Gardner en 1983, publicó Frames of Mind: The theory of multiple intelligenc, postula que el ser humano ha evolucionado para mostrar no solamente una forma de inteligencia flexible sino una variada gama de diferentes inteligencias. Gardner (2006) sostiene que la inteligencia es una "capacidad computacional - una capacidad para procesar cierto tipo de información- originada en la biología y en la psicología humana" (p. 6), 
que es el resultado de la interacción entre factores biológicos y ambientales que dependen de alguna manera del contexto (factores culturales, psicosociales, recursos materiales). Estas capacidades se evidencian en la solución de problemas o en la invención de productos tipificados como valiosos en uno o más entornos culturales o comunidad en particular (Gardner, 1983, 2006). A partir de esta definición se puede inferir que es posible desarrollar la inteligencia, cualidad que está ligada al hecho de que las inteligencias múltiples sean autónomas, pero, al mismo tiempo, cuando se desarrolla la conducta inteligente se requieren de la interacción de dos o más de ellas (Nadal, 2015).

Estas propuestas de Gardner fueron y aún siguen siendo innovadoras en el campo educativo, donde ha recibido la mayor atención e interés, porque brinda un marco teórico diferente para entender el cumplimiento de los propósitos y metas del estudiante (Durán-Aponte, Elvira-Valdés \& Pujol, 2015), pues ellos presentan un perfil característico de inteligencias diferentes que pueden reforzarse con el accionar de profesores competentes, quienes podrán optimizar aquellas inteligencias que se encuentran en un nivel alto y compensar aquéllas que se encuentran en un nivel bajo (Pérez \& Beltrán, 2006). Un punto clave en la teoría considera que "la mayoría de las personas pueden desarrollar todas sus inteligencias hasta conseguir un dominio aceptable de las mismas" (Armstrong, 2006, p.44).

En la Teoría de Gardner la mente está organizada de manera tal que se pueden diferenciar ocho inteligencias o estructuras mentales: lingüística, lógico-matemática, espacial, musical, corporal-cenestésica, naturalista, intrapersonal e interpersonal. Las tres primeras son también denominadas inteligencias académicas, y las dos últimas inteligencias personales (Gardner, 1999). El fundamento de la propuesta de las inteligencias múltiples reposa en un conjunto de variadas informaciones con respaldo fáctico de tipo neurológico (observaciones de pacientes con afecciones del cerebro), evolucionistas (niños excepcionales con retardo mental o preservación de alguna habilidad, o niños y adultos con talentos especiales) y transculturales. 
Gardner, con poco afecto a la medición psicométrica, dio su respaldo al trabajo realizado por Shearer (1995) que construyó y validó el Cuestionario MIDAS cuyo objetivo es medir las inteligencias múltiples. En Perú se elaboró el Cuestionario de Inteligencias Múltiples [CUIM], validado por Aliaga et al. (2012).

Si bien las Teorías de la Autoeficacia y de las Inteligencias Múltiples se elaboraron con propósitos diferentes, comparten dos aspectos comunes; por un lado, ambas están inscritas en el amplio campo del cognitivismo, y por otro, gozan de respaldo empírico puesto que sus deducciones han sido puestas a prueba en diferentes campos de la psicología, especialmente en la psicología educacional. En este campo y muy ligado a la orientación vocacional, Pérez, Beltramino y Cupani (2003) construyeron el Inventario de Autoeficacia para Inteligencias Múltiples (IAMI) compuesto por 69 reactivos, el estudio fue realizado con una muestra de adolescentes de los dos últimos años de educación secundaria en Córdova (Argentina). Luego Pérez y Medrano (2007) realizaron una validación de criterio de este instrumento y un año después, Pérez y Cupani (2008) publicaron una revisión del mismo (IAMI-R) para ser utilizado con fines de orientación vocacional.

El IAMI-R ha sido investigado en relación con otras variables propias del mundo académico. Arias-Gómez y Durán-Aponte (2017) estudiaron con estudiantes venezolanos que se inician en la vida universitaria, la persistencia académica en relación de los factores previos al ingreso universitario, factores motivacionales y experiencias institucionales. Encontraron que estos factores explican en conjunto el 56\% de la variable estudiada. Coballes (2015) relacionó autoconcepto con actividad física, imagen corporal y las inteligencias múltiples, utilizando el IAMI-R, hallaron correlaciones estadísticamente significativas entre las variables mencionadas. Zalazar, Aparicio, Ramírez y Garrido (2011), trabajando con estudiantes argentinos relacionaron una de las escalas del IAMI-R (la autoeficacia lógicomatemática) con una escala de fuentes de autoeficacia lógico matemática y otra de expectativas de resultados en matemáticas, concluyen que estas 
variables presentan correlaciones estadísticamente significativas. Asimismo, Cupani (2010) utilizó una de las escalas del IAMI (lógico-matemática) como criterio de validez predictiva de una escala de expectativas de resultados en matemáticas, encontrando aceptables propiedades psicométricas. Pérez y Medrano (2007) buscaron evidencias de validez de criterio del IAMI-R con el Cuestionario de Intenciones en la Elección de la Carrera (CIEC), sus conclusiones sustentan que el IAMI-R es un instrumento adecuado para usarlo en la orientación vocacional; Pérez, Cupani y Ayllón (2005) estudiaron el rol que juegan las aptitudes, las creencias de autoeficacia y la predicción del rendimiento académico sobre el éxito académico, sus hallazgos confirman el valor de esta predicción.

También se han desarrollado estudios de tipo psicométrico. Entre otros se tiene el estudio de Acosta y Sánchez (2015) analizaron las propiedades psicométricas del IAMI-R con un grupo de estudiantes secundarios colombianos y confirmaron su estructura de ocho factores; ellos eliminaron un ítem de la escala de autoeficacia para la inteligencia interpersonal. A través del coeficiente alfa de Cronbach encontraron valores similares al trabajo original por lo que indican que se trata de un instrumento confiable. DuránAponte, Elvira-Valdés y Pujol (2014) desarrollaron un estudio psicométrico $\mathrm{y}$ validaron el IAMI-R con estudiantes universitarios venezolanos de primer ingreso, a través de un AFC hallaron una estructura de 7 factores, en este trabajo, los autores unieron los factores inter e intrapersonal en uno solo, al que denominaron autoeficacia emocional; acerca de la confiabilidad a través del alfa de Cronbach reportan valores similares a los que comunicaron Pérez y Cupani (2008). Heredia, Pérez, Lescano y Zalazar (2010) construyeron el IAMI-N que se propone identificar el talento de los niños y desarrollar diagnóstico sobre perfiles de aprendizaje de los mismos.

Actualmente se considera que todo proceso de validación de un instrumento debe incluir la validación de constructo, existen cinco fuentes de evidencia de validez, entre ellas la estructura interna del test (American Educational Research Association [AERA], American Psychological 
Association [APA] y National Council on Measurement in Education [NCME], 2014). La forma de obtener estas evidencias tiene que ver con el uso de modelos enraizados en el análisis factorial, entre ellos el modelo de rasgo latente que abarca otros modelos como el del factor común, y los modelos paramétricos y no paramétricos de respuesta al ítem. En este contexto, el propósito del presente estudio es validar el IAMI-R en población peruana de estudiantes de secundaria, siguiendo los procedimientos seguidos por Pérez y Cupani (2008) autores del inventario.

El interés por realizar este estudio se justifica en la calidad de construcción del IAMI-R y en la propuesta de Bandura (2001) para construir escalas de autoeficacia. Duran-Aponte et al. (2014) afirman que es un instrumento bien elaborado, con ítems adecuadamente redactados en tiempo presente, con énfasis en lo que el sujeto puede hacer en situaciones específicas y cuyo contenido representa diferentes grados de desafío o impedimento para un buen desempeño, y que se diferencia de otros instrumentos que evalúan autoeficacia. Igualmente, es justificado el esfuerzo por cuanto éste puede significar un aporte a la psicología peruana, especialmente a aquella dedicada a la educación y la orientación vocacional específicamente. Asimismo, es importante realizar una réplica del estudio original para comprobar su valor en cuanto a la generalización que se le puede otorgar a un instrumento que fue construido según parámetros de la Teoría Clásica de los Tests (TCT), que tiene algunos puntos débiles como la ausencia de invarianza de las propiedades de los ítems y del test respecto de las muestras de los sujetos en las que se le estima (Hambleton \& Van del Liden, 1982).

\section{Método}

\section{Participantes.}

Se trabajó con 1304 participantes, estudiantes de 14 instituciones educativas de la región Lima Metropolitana, que cursaban el quinto año de secundaria de Educación Básica Regular. Con edades comprendidas entre 15 y 19 años, 16.92 años promedio; el 44\% varones, el $60 \%$ pertenecían a instituciones 
educativas estatales. Se trata de una muestra no probabilística, que se compuso por conveniencia, por las facilidades y los permisos que las instituciones educativas otorgaron para aplicar el instrumento, fue dividida en dos muestras del mismo tamaño $(\mathrm{n}=652)$ con la finalidad de aplicar en una de ellas el Análisis Factorial Exploratorio [AFE] y en la otra el Análisis Factorial Confirmatorio [AFC].

\section{Instrumento.}

El Inventario de Autoeficacia para las Inteligencias Múltiples Revisado (IAMI-R) fue publicado por Pérez y Cupani (2008), es una versión nueva del IAMI (Pérez et al., 2003), que fue construido por Pérez (2001) para ser utilizado durante el proceso de orientación vocacional de adolescentes que cursaban el último año de la secundaria, el instrumento tiene como objetivo valorar la autoeficacia o autoconfianza en el desarrollo de actividades académicas asociadas a las inteligencias múltiples, previamente Pérez y Medrano (2007) hicieron un estudio de validez de criterio de este cuestionario.

Pérez y Cupani (2008) trabajaron con 790 estudiantes cordobeses del último año de la escuela media, 59.1\% del sexo femenino, con una edad promedio de 17.31, se les administró una versión de 64 ítems. La muestra fue dividida en dos grupos de 395 estudiantes cada uno. A uno de ellos se le aplicó un AFE, Método de Máxima Probabilidad, con rotación oblicua Promax, identificándose, vía regla de Kayer-Guttman y Scree Plot Test; ocho factores que permitieron explicar el $57.50 \%$ de la varianza. El inventario final tiene 48 ítems que se distribuyen según su contenido y performance psicométricas, cada factor posee seis ítems identificados que se corresponden con las ocho inteligencias propuestas por Gardner y que fueron validadas por jueces (Pérez \& Medrano, 2007). Los resultados de la otra muestra se analizaron a través del AFC, método de Máxima Probabilidad, hallándose que el modelo de ocho factores tenía un ajuste aceptable, pero no óptimo, por lo que los autores consideraron pertinente recomendar la realización de otros estudios semejantes con diferentes muestras (Pérez \& Cupani, 2008). 
Por su lado, los valores del coeficiente alfa de Cronbach son indicadores de una fiabilidad satisfactoria.

Autoeficacia Lingüística. Creencia relacionada con las habilidades para el lenguaje hablado y escrito, el aprendizaje de idiomas y el empleo de la lengua para alcanzar ciertos fines. Ítems del 1 al $6(\alpha=.76)$.

Autoeficacia Lógico-Matemática. Creencia que comprende capacidades para el análisis lógico de problemas, el cálculo numérico y la investigación científica. Ítems 7 al $12(\alpha=.89)$.

Autoeficacia Espacial. Creencia sobre las aptitudes para reconocer y manipular patrones de respuesta amplios y específicos. Ítems del 13 al 18 $(\alpha=.87)$.

Autoeficacia Musical. Creencia que comprende las habilidades para desempeñarse en la ejecución, composición y apreciación con éxito de patrones musicales. Ítems de 19 al $24(\alpha=.92)$.

Autoeficacia Interpersonal. Creencia de poseer habilidades para comprender la personalidad de otros seres humanos y trabajar efectivamente con ellos. Ítems del 25 al $30(\alpha=.79)$.

Autoeficacia Cenestésico-Corporal. Creencia que se relaciona con destrezas para usar el propio cuerpo o parte del mismo en la resolución de problemas o la creación de productos, en específico, lo relacionado con disciplinas deportivas. Ítems del 31 al $36(\alpha=.86)$.

Autoeficacia Intrapersonal. Creencia de comprensión de los propios motivos, sentimientos y capacidades. Ítems del 37 al $42(\alpha=.79)$.

Autoeficacia Naturalista. Creencia relacionada con competencias para el reconocimiento y clasificación de objetos del mundo natural en su ambiente. Ítems del 43 al $48(\alpha=.91)$. 


\section{Procedimiento.}

Contando con la autorización de las instituciones educativas para la administración del instrumento, se coordinó con los docentes de aula y los comités de padres de familia de las aulas que se consideraron disponibles; asimismo, se informó a los alumnos del propósito del estudio, recabándose a través de documentos todos los consentimientos mencionados. Seguidamente, en las propias aulas y en condiciones apropiadas de confort, el equipo encargado de la aplicación del instrumento (estudiantes de psicología del último año de estudios) supervisados por los investigadores y autores del estudio, procedieron a la administración colectiva del IAMI-R.

La información generada se digitó en una base de datos mediante el uso de una hoja de cálculo Microsoft Office Excel. Seguidamente se comprobó la idoneidad de dicha base detectando información incompleta, valores perdidos y/o fuera de rangos (12 casos). Adicionalmente, se identificó y eliminó valores atípicos univariados (14 casos) y multivariados (31 casos), bajo el criterio de distancia de Mahalanobis con punto de corte de $\mathrm{p} \leq .001$ para chi-cuadrado (Tabachnick \& Fidell, 2001).

\section{Análisis de datos}

El análisis psicométrico se realizó con el software libre R, versión 3.5.0 ( $R$ Core Team, 2018) apoyado en los paquetes especializados Psych (Revelle, 2018) para la obtención de los estadísticos descriptivos de los ítems, outliers multivariados y AFE; Lavaan (Rosseel, 2012) para el AFC; y, SemTools (semTools Contributors, 2016) para el análisis de fiabilidad a partir del modelado AFC.

El análisis descriptivo de los ítems se ejecutó calculando el valor de la media, desviación estándar, asimetría y curtosis. La evaluación de los valores de asimetría y curtosis se realizó con vista a precisar su ajuste a la distribución normal empleándose como criterio que sus resultados se encuentren entre 0 y el valor absoluto de 1.50 (Tabahnick \& Fidell, 2001). Con fines de brindar evidencias de validez basada en la estructura interna de 
la prueba mediante análisis factorial, se procedió a generar dos sub-muestras dividiendo por medio de un muestreo aleatorio simple la base de datos en dos bases "A" y "B" ambas con $n=652$ sujetos. En la primera muestra se realizó el análisis factorial exploratorio (AFE) y, en la segunda, el análisis factorial confirmatorio (AFC).

En el proceso del AFE se efectuó los análisis preliminares a su implementación, tales como la medida de adecuación de muestreo KaiserMeyer-Olkin (KMO) y la prueba de esfericidad de Bartlett. Para la estimación de la estructura factorial se empleó el método de Máxima Verosimilitud (MV); asimismo, para precisar el número de factores a retener, se empleó dos procedimientos: la regla de Kaiser-Guttman o Autovalores mayores a 1, y el gráfico de sedimentación (Scree plot test). En cuanto a la rotación de los factores se empleó la rotación oblicua Promax con un parámetro Kappa $=4$ (Hendrickson \& White, 1964).

Con respecto al AFC, se utilizó un método de estimación diferente al MV utilizado por Pérez y Cupani (2008). En efecto, debido a que el IAMI-R es un instrumento de respuestas ordinales, se empleó como insumo a la matriz de correlaciones policóricas (Elosúa \& Zumbo, 2008) con el fin de estimar el ajuste del modelo mediante el método Weighted Least Squares Means and Variance adjusted (WLSMV, Mínimos cuadrados ponderados ajustados) con errores estándar robustos y test estadístico escalado de SS (Scaling-Shifted). Para ello se tuvo en cuenta los índices de ajuste global ( $\chi^{2}$ y RMSEA), índices de ajuste comparativo o incremental robustos (CFI y TLI) e índice de parasimonia ( $\left.\mathrm{SS} \chi^{2} / \mathrm{gl}\right)$. Este análisis fue aplicado bajo la hipótesis que la estructura interna del IAMI-R se ajustaba a un modelo lineal de ocho factores. Se evaluaron dos modelos estadísticos, el primer modelo evalúa la estructura de la prueba especificando los factores latentes de forma relacionada, es decir, correlacionándose entre sí mismos; mientras que el segundo modelo se especifica indicando correlaciones entre todos los factores latentes y la presencia de errores correlacionados entre los ítems de la prueba. 
Por otro lado, siguiendo a la Teoría Clásica de los Tests (TCT), la fiabilidad se fundamenta teóricamente en el concepto de paralelismo: dos tests - o para nuestro caso ítems - son paralelos si presentan igual media y varianza, pero al ser difícil contrastar empíricamente este concepto, se han relajado las condiciones para hacer viable la comprobación empírica de la fiabilidad. En este sentido, el coeficiente de elección, el coeficiente alfa, es mejor interpretable cuando el test es tau equivalente, vale decir, cuando se asume que la varianza del puntaje verdadero es la misma en todos los tests (ítems), pero sin la misma varianza de error. En este estudio, la correlación de los errores de algunos de los ítems, relajan en cierto sentido la tau equivalencia, por lo que fue conveniente también estimar la fiabilidad tratando al test (ítems) como congenérico, es decir asumiendo que las medias y varianzas de los puntajes verdaderos de los tests (ítems) y la varianza de error pueden variar, pero los puntajes verdaderos están linealmente relacionados unos con otros y con la variable latente (Raykov \& Marcoulides, 2011). En otros términos, se asumió que las puntuaciones miden la misma dimensión, factor o rasgo, aunque en diferentes grados y con diferentes errores de medición. En este marco, para el cálculo de la fiabilidad del instrumento se utilizó el método de consistencia interna mediante la estimación del coeficiente Omega de Mc'Donald (Mc Donald, 1999) pues se considera cargas factoriales distintas entre los ítems y diferentes grados de errores en medición de los mismos, pero también se calculó para comparación el coeficiente alfa ordinal.

\section{Resultados}

\section{Consistencia interna.}

El cálculo de los coeficientes alfa ordinal (Contreras \& Novoa-Muñoz, 2018) y Omega de Mc Donald (Mc Donald, 1999) arrojaron resultados bastante similares según se observa en la tabla 6 . Al ser comparados con la gama de intervalos de valores propuestos por George y Mallery (2013): Inaceptable $(<.50)$, pobre (.50 - .60), débil (.60 - .70), aceptable (.70 - .80), bueno ( $.80-.90)$ y excelente $(>.90)$; se observa que la autoeficacia para la inteligencia lógico matemática, tiene el coeficiente más elevado (.945/.946), y la autoeficacia 
para la inteligencia musical, tiene una fiabilidad tipificada de excelente; en tanto que las demás escalas del IAMI-R muestran coeficientes calificados de buenos, el menor es el de la autoeficacia para la inteligencia intrapersonal (.859/.857). En síntesis: a partir de los tamaños de los coeficientes Alfa ordinal y Omega de Mc Donald de las escalas del IAMI-R, en la muestra de estudiantes peruanos del quinto año de educación secundaria, se puede entender que estos dan respuestas semejantes a ítems que son diferentes pero que manifiestan conceptualmente la variable latente medida.

\section{Resultados descriptivos.}

En la Tabla 1 se muestra el análisis descriptivo de los ítems, entre los valores de la media aritmética se observa que seis ítems tienen medias ligeramente superiores a 7 (marcadas con negritas) en la escala que va del 1 al 10, la media de los 42 ítems restantes fluctúa por debajo de 7 hasta 4; el ítem 42 (identificar rápidamente tus emociones) tiene la media más elevada, en tanto que, el ítem 22 (afinar un instrumento musical) tiene la media más baja. Respecto a la desviación estándar, sus valores varían de 2.06 a 2.78, teniendo la desviación más alta el ítem 23 (tocar un instrumento en una orquesta o grupo musical), y el ítem 41 la más baja (conocer tus capacidades para enfrentar diferentes situaciones). Finalmente, los valores de asimetría y curtosis indican que 40 ítems tienen valores en el rango $+1 /-1$, que es un resultado calificado de excelente, mientras que en los otros 8 se tiene un valor máximo de -1.12, evaluado como adecuado (George \& Mallery, 2013). Estos resultados sugieren que las distribuciones de los ítems del IAMI-R, se ajustan a un patrón de distribución normal. 


\section{Tabla 1.}

Análisis descriptivo, asimetría y curtosis de los ítems del IAMI-R $(n=1304)$.

\begin{tabular}{|c|c|c|c|c|c|c|}
\hline & Escala & Ítem & M & $\mathrm{DE}$ & Asimetría & Curtosis \\
\hline & Lingüística & 01 & 5.65 & 2.34 & 0.07 & -0.78 \\
\hline & & 02 & 4.96 & 2.18 & 0.35 & -0.46 \\
\hline & & 03 & 5.77 & 2.25 & -0.03 & -0.78 \\
\hline & & 04 & 5.16 & 2.32 & 0.21 & -0.73 \\
\hline & & 05 & 5.81 & 2.32 & -0.08 & -0.77 \\
\hline & & 06 & 5.59 & 2.41 & 0.01 & -0.84 \\
\hline \multirow[t]{6}{*}{ A. } & $\begin{array}{l}\text { Lógico- } \\
\text { Matemática }\end{array}$ & 07 & 5.14 & 2.50 & 0.14 & -0.85 \\
\hline & & 08 & 5.76 & 2.67 & -0.04 & -1.02 \\
\hline & & 09 & 5.51 & 2.60 & 0.06 & -1.01 \\
\hline & & 10 & 5.64 & 2.57 & -0.02 & -0.94 \\
\hline & & 11 & 6.08 & 2.65 & -0.19 & -1.00 \\
\hline & & 12 & 5.81 & 2.58 & -0.07 & -0.96 \\
\hline & Espacial & 13 & 5.70 & 2.69 & -0.06 & -1.08 \\
\hline & & 14 & 5.92 & 2.59 & -0.13 & -0.95 \\
\hline & & 15 & 5.57 & 2.53 & -0.01 & -0.92 \\
\hline & & 16 & 5.57 & 2.51 & -0.05 & -0.83 \\
\hline & & 17 & 5.58 & 2.53 & 0.00 & -0.90 \\
\hline & & 18 & 5.31 & 2.69 & 0.07 & -1.03 \\
\hline \multirow{6}{*}{\multicolumn{2}{|c|}{ A. Musical }} & 19 & 5.99 & 2.83 & -0.20 & -1.12 \\
\hline & & 20 & 4.88 & 2.78 & 0.28 & -1.02 \\
\hline & & 21 & 4.82 & 2.77 & 0.32 & -1.02 \\
\hline & & 22 & 4.13 & 2.66 & 0.58 & -0.69 \\
\hline & & 23 & 4.68 & 2.96 & 0.33 & -1.12 \\
\hline & & 24 & 4.46 & 2.72 & 0.46 & -0.83 \\
\hline & Interpersonal & 25 & 7.09 & 2.22 & -0.65 & -0.16 \\
\hline & & 26 & 6.63 & 2.24 & -0.39 & -0.43 \\
\hline & & 27 & 6.21 & 2.55 & -0.23 & -0.92 \\
\hline & & 28 & 7.07 & 2.29 & -0.58 & -0.33 \\
\hline & & 29 & 6.95 & 2.36 & -0.54 & -0.43 \\
\hline
\end{tabular}




\begin{tabular}{|c|c|c|c|c|c|c|}
\hline \multirow{7}{*}{ A. } & & 30 & 7.13 & 2.29 & -0.58 & -0.37 \\
\hline & \multirow[t]{6}{*}{$\begin{array}{l}\text { Cenestésico- } \\
\text { Corporal }\end{array}$} & 31 & 6.65 & 2.50 & -0.45 & -0.67 \\
\hline & & 32 & 6.38 & 2.41 & -0.30 & -0.70 \\
\hline & & 33 & 6.08 & 2.76 & -0.20 & -1.05 \\
\hline & & 34 & 6.27 & 2.73 & -0.30 & -0.97 \\
\hline & & 35 & 6.27 & 2.61 & -0.29 & -0.90 \\
\hline & & 36 & 5.88 & 2.51 & -0.18 & -0.81 \\
\hline \multirow[t]{6}{*}{ A. } & Intrapersonal & 37 & 6.94 & 2.18 & -0.54 & -0.23 \\
\hline & & 38 & 6.76 & 2.71 & -0.60 & -0.63 \\
\hline & & 39 & 6.88 & 2.28 & -0.55 & -0.30 \\
\hline & & 40 & 7.26 & 2.18 & -0.65 & -0.18 \\
\hline & & 41 & 7.20 & 2.06 & -0.66 & 0.15 \\
\hline & & 42 & 7.53 & 2.13 & -0.76 & 0.07 \\
\hline \multirow[t]{6}{*}{ A. } & Naturalista & 43 & 5.57 & 2.60 & -0.05 & -0.94 \\
\hline & & 44 & 5.16 & 2.44 & 0.12 & -0.83 \\
\hline & & 45 & 4.74 & 2.49 & 0.31 & -0.77 \\
\hline & & 46 & 4.82 & 2.56 & 0.27 & -0.82 \\
\hline & & 47 & 4.64 & 2.59 & 0.34 & -0.86 \\
\hline & & 48 & 5.85 & 2.55 & -0.13 & -0.84 \\
\hline
\end{tabular}

\section{Análisis Factorial Exploratorio (AFE).}

El índice KMO tuvo un valor de 926 tipificado como excelente (García, Gil \& Rodríguez, 2000), en tanto que la prueba de esfericidad de Bartlett entregó un resultado $X^{2}$ estadísticamente muy significativo (23085.258, p < .001). Aplicado el método de Máxima Verosimilitud (MV), se identificó una solución de ocho factores, realizándose seguidamente una rotación Promax, debido a que en varios ítems se presentaron intercorrelaciones mayores a 0.32 (tabla 2) (Tabachnick \& Fidell, 2001). Intercorrelaciones que, por otra parte, son distantes de .80 valor propuesto como indicador de colinealidad (Berry \& Feldman, como se citó en Cea, 2004). 


\section{Tabla 2.}

Intercorrelaciones entre los factores (AFE) $\left(n_{A}=652\right)$.

\begin{tabular}{lcccccccc}
\hline \multicolumn{1}{c}{ Ítem } & F-I & F-II & F-III & F-IV & F-V & F-VI & F-VII & F-VIII \\
\hline F-I & - & & & & & & & \\
F-II & .176 & - & & & & & & \\
F-III & $\mathbf{. 3 5 1}$ & $\mathbf{. 3 6 5}$ & - & & & & & \\
F-IV & $\mathbf{. 3 8 8}$ & $\mathbf{. 3 4 7}$ & $\mathbf{. 4 4 3}$ & - & & & & \\
F-V & .246 & .299 & $\mathbf{. 4 3 5}$ & .278 & - & & & \\
F-VI & $\mathbf{. 3 7 5}$ & .280 & .287 & .312 & $\mathbf{. 4 8 8}$ & - & & \\
F-VII & .285 & $\mathbf{. 4 2 4}$ & $\mathbf{. 4 7 7}$ & $\mathbf{. 4 2 2}$ & $\mathbf{. 4 1 9}$ & $\mathbf{. 4 1 8}$ & - & \\
F-VIII & $\mathbf{. 3 3 4}$ & $\mathbf{. 3 9 0}$ & $\mathbf{. 4 5 6}$ & .294 & $\mathbf{. 6 5 5}$ & $\mathbf{. 5 4 5}$ & $\mathbf{. 4 5 0}$ & - \\
\hline
\end{tabular}

La rotación Promax determinó que los pesos factoriales de los ítems, todos superiores a .30, corresponden al ordenamiento teórico del IAMI-R, en consecuencia, el primer factor fue etiquetado como autoeficacia para la inteligencia lingüística, el segundo autoeficacia para la inteligencia lógico-matemática, el tercero autoeficacia para la inteligencia espacial, el cuarto autoeficacia para la inteligencia musical, el quinto autoeficacia para la inteligencia interpersonal, el sexto autoeficacia para la inteligencia cenestésica corporal, el sétimo autoeficacia para la inteligencia intrapersonal, y el octavo autoeficacia para la inteligencia naturalista. 
Tabla 3.

Análisis Factorial Exploratorio, Rotación Promax, Matriz de estructura $\left(n_{A 652}\right)$.

\begin{tabular}{|c|c|c|c|c|c|c|c|c|c|}
\hline Ítem & ALING & ALOMA & AESPA & AMUSI & AINTER & ACENE & AINTRA & ANATU & $\mathrm{h}^{2}$ \\
\hline 01 & .560 & & & & & & & & .405 \\
\hline 02 & .608 & & & & & & & & .423 \\
\hline 03 & .578 & & & & & & & & .471 \\
\hline 04 & .935 & & & & & & & & .751 \\
\hline 05 & .710 & & & & & & & & .600 \\
\hline 06 & .859 & & & & & & & & .670 \\
\hline 07 & & .633 & & & & & & & .491 \\
\hline 08 & & .904 & & & & & & & .768 \\
\hline 09 & & .883 & & & & & & & .791 \\
\hline 10 & & .886 & & & & & & & .819 \\
\hline 11 & & .925 & & & & & & & .846 \\
\hline 12 & & .930 & & & & & & & .864 \\
\hline 13 & & & .806 & & & & & & .605 \\
\hline 14 & & & .851 & & & & & & .705 \\
\hline 15 & & & .935 & & & & & & .787 \\
\hline 16 & & & .792 & & & & & & .675 \\
\hline 17 & & & .651 & & & & & & .538 \\
\hline 18 & & & .673 & & & & & & .511 \\
\hline 19 & & & & .737 & & & & & .622 \\
\hline 20 & & & & .686 & & & & & .538 \\
\hline 21 & & & & .751 & & & & & .595 \\
\hline 22 & & & & .870 & & & & & .746 \\
\hline 23 & & & & .885 & & & & & .734 \\
\hline 24 & & & & .859 & & & & & .707 \\
\hline 25 & & & & & .500 & & & & .474 \\
\hline 26 & & & & & .723 & & & & .635 \\
\hline 27 & & & & & .583 & & & & .488 \\
\hline 28 & & & & & .763 & & & & .612 \\
\hline 29 & & & & & .725 & & & & .637 \\
\hline 30 & & & & & .715 & & & & .589 \\
\hline 31 & & & & & & .610 & & & .521 \\
\hline 32 & & & & & & .617 & & & .586 \\
\hline
\end{tabular}




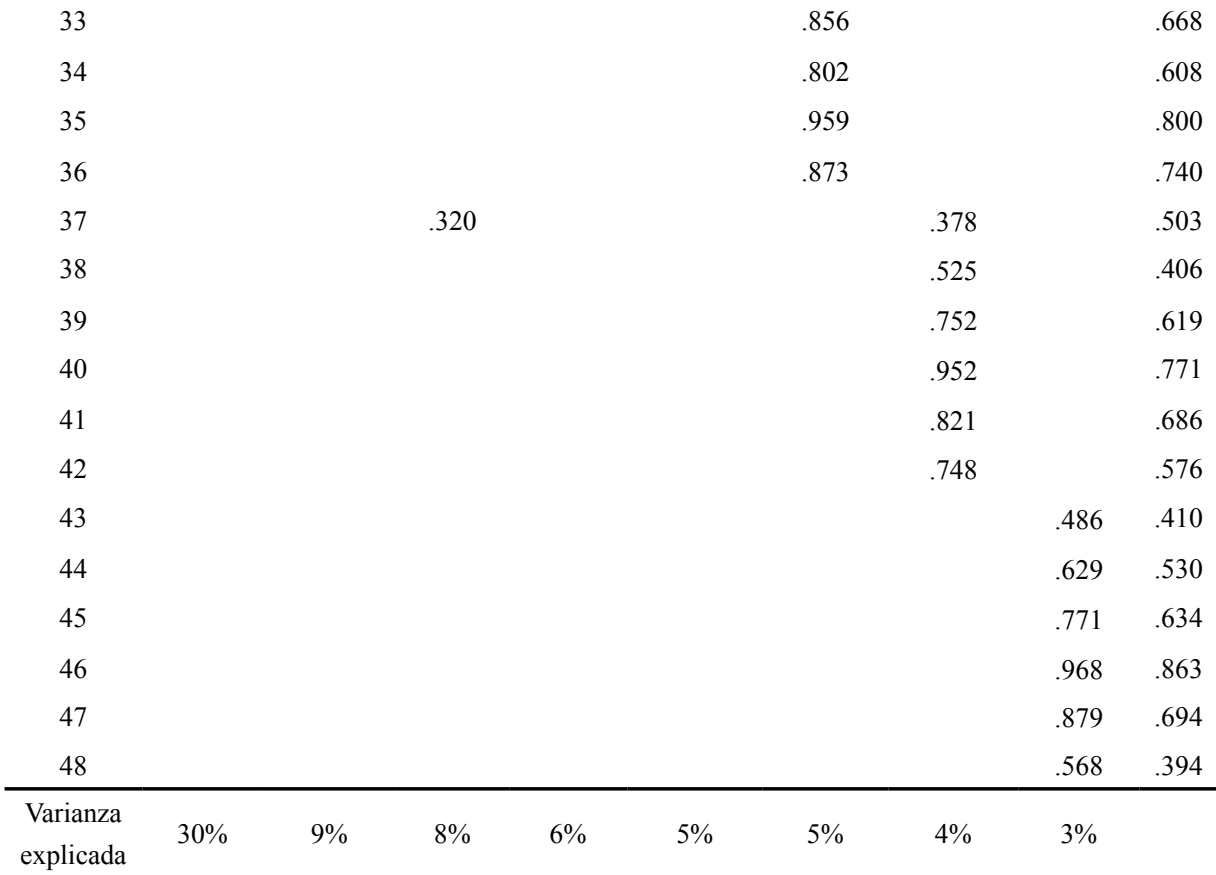

Nota . ALING = Autoeficacia para la inteligencia lingüística, ALOMA = Autoeficacia para la inteligencia lógico-matemática; AESPA = Autoeficacia para la inteligencia espacial; AMUSI = Autoeficacia para la inteligencia musical; AINTER = Autoeficacia para la inteligencia Interpersonal; ACENE = Autoeficacia para la inteligencia cenestésica-corporal; AINTRA = Autoeficacia para la inteligencia intrapersonal; ANATU $=$ Autoeficacia para la inteligencia naturalista.

En la Tabla 3 sólo aparecen las cargas o saturaciones (loading) mayores a .30. En el caso del ítem 37 que cargaba en autoeficacia para la inteligencia espacial y en autoeficacia para la inteligencia intrapersonal, se determinó agruparla en esta última en vista que allí tenía la carga más elevada. Asimismo, puede inferirse que el promedio de las cargas en todos los factores es superior a .50 lo cual denota factores bien definidos (Comrey, 1985), los mismos que explican el 70\% de la varianza de las puntuaciones. 
Tabla 4.

Estadísticos descriptivos de los factores $\left(n_{A}=652\right)$.

\begin{tabular}{lcccc}
\hline \multicolumn{1}{c}{ Factor $(*)$} & M & DE & Asimetría & Curtosis \\
\hline ALING & 33.94 & 13.82 & -0.01 & -0.88 \\
ALOMA & 28.96 & 14.11 & 0.30 & -0.77 \\
AESPA & 37.52 & 12.82 & -0.23 & -0.72 \\
AMUSI & 33.66 & 12.78 & 0.02 & -0.74 \\
AINTER & 42.57 & 10.57 & -0.46 & -0.20 \\
ACENE & 32.93 & 10.76 & 0.07 & -0.53 \\
AINTRA & 30.79 & 12.11 & 0.19 & -0.60 \\
ANATU & 41.07 & 11.02 & -0.37 & -0.44 \\
\hline
\end{tabular}

(*) ALING = Autoeficacia para la inteligencia lingüística, ALOMA = Autoeficacia para la inteligencia lógico-matemática; AESPA = Autoeficacia para la inteligencia espacial; AMUSI = Autoeficacia para la inteligencia musical; AINTER = Autoeficacia para la inteligencia Interpersonal; ACENE = Autoeficacia para la inteligencia cenestésica-corporal; AINTRA = Autoeficacia para la inteligencia intrapersonal; ANATU $=$ Autoeficacia para la inteligencia naturalista.

En la Tabla 4, se observa que la autoeficacia para la inteligencia interpersonal tiene la media más alta, seguidas por autoeficacia para la inteligencia naturalista, autoeficacia para la inteligencia espacial, autoeficacia para la inteligencia lingüística y autoeficacia para la inteligencia musical. A continuación se sitúan autoeficacia para la inteligencia cenestésica-corporal, autoeficacia para la inteligencia naturalista y, al último, autoeficacia para la inteligencia lógico-matemática tiene la media más baja y, a su vez, la dispersión más grande, en tanto que la dispersión más pequeña corresponde a autoeficacia para la inteligencia interpersonal. Por otra parte, los valores de asimetría y curtosis de los factores se encontraron entre $+/-1$, lo que indica que sus distribuciones son normales (George \& Mallery, 2013).

\section{Análisis factorial confirmatorio (AFC)}

Este segundo modelo considera la correlación de los errores entre los ítems 4 y 6 (autoeficacia para la inteligencia lingüística); 13, 14 y 15 (autoeficacia para la inteligencia espacial); 35 (autoeficacia para la inteligencia cenestésica 
corporal) y 45 (autoeficacia para la inteligencia naturalista), debido a la presencia de índices de modificación que lo sugieren y la detección de redacción similar al inicio del ítem y también su referencia a conceptos lingüísticos que pueden haber sido confundidos por los sujetos, por ejemplo el ítem 4 dice: "Escribir una redacción breve sin errores gramaticales", mientras que el ítem 6 dice: "Escribir una redacción breve sin errores ortográficos". Los resultados se muestran en la Tabla 5.

\section{Tabla 5.}

Índices de ajustes de dos modelos de medición en IAMI-R en la muestra peruana $\left(n_{B}=652\right)$.

\begin{tabular}{cccccccc}
\hline Modelo & $\mathrm{SS} \chi^{2}$ & $\mathrm{gl}$ & $\mathrm{SS} \chi^{2} / \mathrm{g} 1$ & $\begin{array}{c}\text { RMSEA } \\
{[\mathrm{IC} 90 \%]}\end{array}$ & SRMR & CFI & TLI \\
\hline Modelo 1 & 1950.11 & 1052 & 1.85 & $\begin{array}{c}.037 \\
{[.035, .040]}\end{array}$ & .050 & .906 & .899 \\
Modelo 2 & 1857.73 & 1047 & 1.77 & $\begin{array}{c}.035 \\
.033, .038]\end{array}$ & .049 & .915 & .908 \\
\hline
\end{tabular}

$\mathrm{SS} \chi^{2}=$ chi-cuadrado ajustado; $\mathrm{gl}=$ grados de libertad; $\mathrm{SS} \chi^{2} / \mathrm{gl}=$ índice de parsimonia; RMSEA= error cuadrado de aproximación a las raíces medias; SRMR= Raíz del residuo cuadrático promedio estandarizado; CFI = índice de ajuste comparativo; TLI = índice de Tucker Lewis.

El índice de ajuste global RMSEA tiene en ambos modelos un valor inferior a .05 indicando un buen ajuste a los datos, afirmación que también se sustenta en el hecho que el intervalo de confianza al 90\% (I.C) se ubica entre 0 y .05 (Schumacker \& Lomax, 2016), lo mismo sucede con el índice SRMR. Por otro lado, los índices de ajuste comparativo señalan que en el CFI ambos modelos tienen valores por encima de .90, valor aceptable según Hu y Bentler (1999), en tanto que el modelo 2 también cumple esta especificación en TLI, no así el modelo 1. Asimismo, respecto al índice de parsimonia $\mathrm{SS} X^{2} / \mathrm{gl}$, ambos modelos tienen valores inferiores a 3, que es lo adecuado (Marsh \& Hocevar, 1985), pero el modelo 2 presenta una mejor relación entre el valor de chi-cuadrado ajustado y sus grados de libertad. Finalmente la observación conjunta de los valores de los indicadores estadísticos utilizados muestra que 
el modelo 2, tiene un mejor ajuste global satisfactorio en la comprobación de la estructura interna del IAMI-R de ocho factores o autoeficacias para las inteligencias múltiples en estudiantes de quinto año de secundaria de educación básica regular de Lima Metropolitana, pero no debe perderse de vista la existencia de errores correlacionados en algunos de los ítems pertenecientes a algunas de las escalas del IAMI-R anotadas anteriormente, que estarían afectando su fiabilidad.

La Tabla 6 muestra los pesos factoriales estandarizados del AFC del segundo modelo. Se observa que la menor carga factorial estandarizada es de 0.573 correspondiente al ítem 3 de la dimensión autoeficacia para la inteligencia espacial, mientras que la mayor carga es de 0.902 perteneciente al ítem 9 de la dimensión autoeficacia para la inteligencia lógico matemática. Por otro lado, se encontró que las correlaciones entre los factores fluctúan entre 0.134 (autoeficacia para la inteligencia lógico matemático y autoeficacia para la inteligencia musical), y 0.712 (autoeficacias para la inteligencia interpersonal e intrapersonal).

\section{Tabla 6.}

AFC. Saturaciones de los items en el modelo 2 del IAMI-R $\left(n_{B}=652\right)$.

\begin{tabular}{|c|c|c|c|c|c|c|c|c|}
\hline Ítem & ALING & ALOMA & AESPA & AMUSI & AINTER & ACENE & AINTRA & ANATU \\
\hline 01 & .726 & & & & & & & \\
\hline 02 & .691 & & & & & & & \\
\hline 03 & .739 & & & & & & & \\
\hline 04 & .667 & & & & & & & \\
\hline 05 & .741 & & & & & & & \\
\hline 06 & .672 & & & & & & & \\
\hline 07 & & .812 & & & & & & \\
\hline 08 & & .855 & & & & & & \\
\hline 09 & & .902 & & & & & & \\
\hline 10 & & .880 & & & & & & \\
\hline 11 & & .864 & & & & & & \\
\hline 12 & & .856 & & & & & & \\
\hline 13 & & & .573 & & & & & \\
\hline 14 & & & .762 & & & & & \\
\hline
\end{tabular}




\begin{tabular}{|c|c|c|c|c|c|c|c|c|}
\hline 15 & & & .715 & & & & & \\
\hline 16 & & & .818 & & & & & \\
\hline 17 & & & .832 & & & & & \\
\hline 18 & & & .753 & & & & & \\
\hline 19 & & & & .790 & & & & \\
\hline 20 & & & & .765 & & & & \\
\hline 21 & & & & .838 & & & & \\
\hline 22 & & & & .844 & & & & \\
\hline 23 & & & & .802 & & & & \\
\hline 24 & & & & .855 & & & & \\
\hline 25 & & & & & .675 & & & \\
\hline 26 & & & & & .816 & & & \\
\hline 27 & & & & & .740 & & & \\
\hline 28 & & & & & .730 & & & \\
\hline 29 & & & & & .777 & & & \\
\hline 30 & & & & & .717 & & & \\
\hline 31 & & & & & & .740 & & \\
\hline 32 & & & & & & .833 & & \\
\hline 33 & & & & & & .721 & & \\
\hline 34 & & & & & & .749 & & \\
\hline 35 & & & & & & .760 & & \\
\hline 36 & & & & & & .785 & & \\
\hline 37 & & & & & & & .741 & \\
\hline 38 & & & & & & & .583 & \\
\hline 39 & & & & & & & .778 & \\
\hline 40 & & & & & & & .715 & \\
\hline 41 & & & & & & & .800 & \\
\hline 42 & & & & & & & .674 & \\
\hline 43 & & & & & & & & .707 \\
\hline 44 & & & & & & & & .714 \\
\hline 45 & & & & & & & & .786 \\
\hline 46 & & & & & & & & .753 \\
\hline 47 & & & & & & & & .775 \\
\hline 48 & & & & & & & & .716 \\
\hline Alfa & .865 & .945 & .899 & .922 & .880 & .899 & .859 & .891 \\
\hline Omega & .833 & .946 & .824 & .922 & .881 & .880 & .857 & .892 \\
\hline
\end{tabular}




\section{Discusión}

La autoeficacia y las inteligencias múltiples son constructos de raigambre cognitiva que desde su elaboración (Bandura, 1997; Gardner, 1983) despertaron el interés de psicólogos y educadores latinoamericanos por su valor teórico y práctico para el análisis, explicación y predicción de comportamientos que se ponen de manifiesto en el mundo académico, entre ellos el rendimiento académico, la persistencia académica, el desempeño docente, la orientación a metas académicas, la orientación vocacional (Alegre, 2014; Aliaga et al, 2012, Arias-Gómez \& Durán-Aponte,2017; Covarrubias \& Mendoza, 2013; Olaz ,2001; Pérez et al, 2005). Teniendo en cuenta estas bondades, Pérez, Beltramino y Cupani (2003) con base en los marcos conceptuales de la teoría de las inteligencias múltiples de Gardner (1983) y de la teoría social-cognitiva de Bandura (1997) y en un modelo social cognitivo de desarrollo de carrera propuesto por Olaz (2003), crearon para su uso en orientación vocacional el IAMI un instrumento que operativamente unifico ambos constructos con la finalidad de medir la confianza de los estudiantes argentinos que finalizaban la secundaria en el proceso de efectuar con éxito actividades vinculadas con las ocho inteligencias múltiples. El 2008 el instrumento fue revisado y adecuado psicométricamente para su uso en estudiantes secundarios más jóvenes, versión conocida como IAMI-R. En este marco, este estudio de carácter psicométrico e instrumental tuvo como propósito establecer la validez y fiabilidad del IAMI-R en estudiantes peruanos que cursan el quinto año de secundaria en colegios estatales y privados de Lima Metropolitana, siguiendo un procedimiento lo más cercano posible al que siguieron los autores de la prueba.

Los resultados descriptivos indican que todos los ítems del instrumento tienen buenas características psicométricas, asimismo, que los resultados obtenidos mediante el AFE y el AFC indican que en la muestra de adolescentes peruanos se halla y confirma la estructura interna de ocho factores del IAMI-R encontrada en el estudio original realizado por Pérez y Cupani (2008) con una muestra de adolescentes argentinos de la ciudad 
de Córdova; ratificado por los datos obtenidos en el estudio efectuado por Acosta y Sánchez (2015) con estudiantes colombianos que cursan similar año de instrucción secundaria. Esta replica permite sostener que existen evidencias empíricas suficientes de generalización de la validez del IAMI-R en referencia a esta estructura para su uso como instrumento medidor de la autoeficacia para las inteligencias múltiples en estudiantes latinoamericanos que finalizan sus estudios secundarios. El proceso seguido es sólido y robusto lo cual hace legítimos sus resultados, sin embargo, por las características de la TCT que es la teoría psicométrica en la que se fundamenta la construcción del IAMI-R es conveniente realizar estudios adicionales que abarquen otras de las fuentes de validez no solamente para su empleo en el campo de la orientación vocacional que fue el interés primigenio de sus autores, sino también para otros propósitos en el ámbito de la psicología educativa y potencialmente en otros contextos no educativos.

Existen también otros estudios realizados con el IAMI-R que no han validado los ocho factores sino siete, integrando los factores de inteligencia intrapersonal e interpersonal en un solo factor, cuestión que interpretativamente tampoco genera muchas diferencias de tipo conceptual (Durán-Aponte et al., 2014; Pérez \& Medrano, 2013) y que de hecho se han integrado en el concepto de inteligencia emocional que se viene trabajando a partir de Goleman (1995)

Por otra parte, teniendo en cuenta que la correlación de los errores en algunos pocos ítems implica el análisis de su formulación, la medición de los constructos subyacentes a las escalas del inventario puede hacerse con una buena precisión ya que las puntuaciones de los adolescentes peruanos mostraron unos índices apropiados de fiabilidad en su modalidad de consistencia interna tanto en su cálculo vía coeficiente alfa ordinal como en su cálculo vía coeficiente de McDonald, incluso algunos de los índices hallados son más elevados que los obtenidos en los adolescentes argentinos por Pérez y Cupani (2008), siendo valorados como indicadores de una fiabilidad que 
va de buena (0.865/0.833 Autoeficacia lingüística), a excelente $(0.945 / 0.946$ Autoeficacia lógico-matemática) (George y Mallery, 2013).

En consecuencia, en relación al propósito del estudio se puede concluir que se establece la validez del IAMI-R mediante el análisis de su estructura interna vía AFE y AFC encontrándose además evidencias de la generalización de su estructura de ocho factores tanto en estudiantes argentinos, colombianos y peruanos que están acabando la educación secundaria. Asimismo, la fiabilidad del IAMI-R queda asegurada pues los índices de fiabilidad alfa ordinal y Omega de McDonald tienen cuantías de tamaño suficiente para medir con precisión las ocho autoeficacias para las inteligencias múltiples en los estudiantes peruanos.

Finalmente, este trabajo representa un aporte para el quehacer profesional del psicólogo educativo peruano, respecto de la medición de variables que tienen impacto en el desempeño académico del estudiante y en aplicaciones en diferentes actividades del psicólogo escolar. Estos resultados así como de otras realizadas con el IAMI-R confirman que ella es útil tanto para ser utilizada en la orientación y asesoramiento vocacional con estudiantes que están en el proceso de elegir una carrera como, como para ser utilizada en la elección de una especialidad en los estudios universitarios y también cuando se desea realizar orientación académica de los estudiantes a partir un perfil cognitivo (como un diagnóstico de sus fortalezas y debilidades autopercibidas); del mismo modo, podría ser útil para desarrollar potencialidades cognitivas a través de programas de intervención.

Un hallazgo interesante derivado de la estructura factorial del IAMI-R es que salvo alguna diferencia en el orden tanto en la investigación de Pérez y Cupani (2003) como en el de Acosta y Sánchez (2015) y el presente estudio, los factores que explican la mayor cantidad de varianza son la autoeficacia lingüística, la autoeficacia lógico-matemática y la autoeficacia espacial, que en el caso de las inteligencias múltiples Gardner (1999) denomina como las inteligencias académicas. Posiblemente este hallazgo se deba a que la población analizada sea de escolares secundarios. Pero estas conjeturas, como 
los perfiles que pueden desarrollar escolares de nivel primario, secundario y universitario, y las necesarias comparaciones por sexo, pueden ser parte de los problemas de posteriores investigaciones.

\section{Referencias}

Acosta-Amaya, M., \& Sánchez-Escudero, J.(2015). Desempeño psicométrico de dos escalas de autoeficacia e intereses profesionales en una muestra de estudiantes de secundaria. Revista CES Psicología, 8(2), 156-170.

Alarcón, R. (2008). Métodos y Diseños de Investigación del Comportamiento. Lima: Editorial Universitaria, Universidad Ricardo Palma.

Alegre, A. (2014). Autoeficacia académica, autorregulación del aprendizaje y rendimiento académico en estudiantes universitarios iniciales. Propósitos $y$ representaciones, 2(1), 79-120. Doi: http://dx.doi.org/10.20511/ pyr2014.v2n1.54

Aliaga, J., Ponce, C., Bulnes, M., Elizalde, R., Montgomery, W., et al. (2012). Las inteligencias múltiples: evaluación y relación con el rendimiento en matemática en estudiantes del quinto año de secundaria de Lima Metropolitana. Revista IIPSI, 15(2), 163-202.

American Educational Research Association, American Psychological Association \& National Council on Measurement in Education (2014). Standards for educational and psychological testing. Washington, DC: American Educational Research Association.

Amstrong, T. (2006). Inteligencias múltiples en el aula. Guía práctica para educadores. Barcelona: Paidós Ibérica.

Arias-Gómez, D., \& Durán-Aponte, E. (2017). Persistencia académica en un programa de nivelación universitario venezolano: caso Universidad Simón Bolívar. Revista Digital de Investigación en Docencia Universitaria, 11(2), 289-307. Doi: https://doi.org/10.19083/ridu.11.512

Ato, M., López, J., \& Benavente, A. (2013). Un sistema de clasificación de los diseños de investigación en psicología. Anales de Psicología, 29(3), 1038-1059. Doi: https://doi.org/10.6018/analesps.29.3.178511

Bandura, A. (1977a). Social learning theory. Englewood Cliffs, NJ: Prentice-Hall. 
Bandura, A. (1977b). Self-efficacy: Toward a unifying theory of behavioral change. Psychological Review, 84, 191-215. Doi: https://doi. org/10.1037/0033-295X.84.2.191

Bandura, A. (1986). Social foundations of thought and action. A social cognitive theory. Englewood Cliff, NJ: Prentice Hall.

Bandura, A. (1995). Self-efficacy in changing societies. Cambridge: University Press. Doi: https://doi.org/10.1017/CBO9780511527692

Bandura, A. (1997). Self-efficacy: The exercise of control. New York: W. H. Freeman.

Bandura, A. (2001). Guía para la construcción de escalas de autoeficacia. Recuperado de https://www.uky.edu/ eushe2/Pajares/effguideSpanish.html

Cea de Ancona, Ma. (2004). Análisis Multivariable. Teoría y Práctica en la Investigación Social. Madrid: Síntesis.

Coballes, S. (2015). Relaciones entre actividad fisica, imagen corporal, autoconcepto e inteligencias múltiples de los adolescentes (Tesis de maestría). Universidad de Oviedo, España. Recuperado de: http://hdl. handle.net/10651/37417

Contreras Espinoza, S., \& Novoa-Muñoz, F. (2018). Ventajas del alfa ordinal respecto al alfa de Cronbach ilustradas con la encuesta AUDIT-OMS. Revista Panamericana de Salud Pública. 42, 1-6. Doi: https://doi. org/10.26633/RPSP.2018.65

Comrey, A. L. (1985) Manual de análisis factorial. Madrid: Cátedra.

Covarrubias, C., \& Mendoza, M. (2013). La teoría de autoeficacia y el desempeño docente: el caso de Chile. Estudios Hemisféricos y Polares, 4(2), 107-123.

Cupani, M. (2010). Validación de una nueva escala de expectativas de resultados y metas de rendimiento para Matemática. Interdisciplinaria, 27(1), 111-127.

Durán-Aponte, E., Elvira-Valdés, M., \& Pujol, L. (2014). Validación del Inventario de Autoeficacia para Inteligencias Múltiples Revisado (IAMI-R) en una muestra de estudiantes universitarios venezolanos. Revista Electrónica Actualidades Investigativas en Educación, 14(2), 1-23.

Durán-Aponte, E., Elvira-Valdés, M. \& Pujol, L. (2015). Orientación a las metas académicas, persistencia y rendimiento en estudiantes del Ciclo de Iniciación Universitaria. REDU, Revista de Docencia Universitaria, 13(2), 189-205 Doi: https://doi.org/10.4995/redu.2015.5444 
Gálvez, E., Chia, N., \& Valdez, J. (2005). Género, ansiedad social, autoeficacia general y autoeficacia en situaciones sociales. Revista de Psiquiatría y Salud Mental Hermilio Valdizan, 6(1), 43-54.

García, E., Gil, J., \& Rodríguez, G. (2000). Análisis factorial. Madrid: La Muralla Herpérides.

Gardner, H. (1983). Frames of Mind: The theory of multiple intelligence. New York: Basic Books.

Gardner, H. (1999). Intelligence reframe: Multiple intelligences for the 21st century. New York: Basic Books.

Gardner H. (2006). Múltiple Intelligences New Horizons. New York : Basic Books.

George, D., \& Mallery, P. (2013). IBM SPSS Statistics 21 step by step: a simple guide and reference (13a ed.). Boston: Pearson.

Goleman, D. (1995). Inteligencia Emocional. España: Editorial Kairos

Hambleton, R., \& Van der Linden, W. (1982). Advances in ítem response theory and applications: An introduction. Applied Psychological Measurement, 6(4), 373-378. Doi: https://doi.org/10.1177/014662168200600401

Hendrickson,A.E., \& White, P. O.(1964). Promax:Aquick method for rotation to oblique simple structure. British Journal of Statistical Psychology, 17(1), 65-70. Doi: https://doi.org/10.1111/j.2044-8317.1964.tb00244.x

Heredia, D. E., Perez, E., Lescano, C. V. \& Zalazar, M. P. (2010). Construcción de un inventario de autoeficacia para inteligencias múltiples en niños (IAMI-N). II Congreso Internacional de Investigación y Práctica Profesional en Psicología XVII Jornadas de Investigación Sexto Encuentro de Investigadores en Psicología del MERCOSUR. Facultad de Psicología - Universidad de Buenos Aires.

Hu, L., \& Bentler, P. M. (1999). Cut off criteria for fit 36 indexes in covariance structure analysis: Conventional criteria versus new alternatives. Structural Equation Modeling, 6(1), 1-55. Doi: https://doi. org/10.1080/10705519909540118

Lazarus, R., \& Folkman, S. (1986). Estrés y procesos cognitivos. Barcelona: Martínez Roca

McDonald, R. P. (1999). Test theory: A unified treatment. Mahwah: Lawrence Erlbaum Associates, Inc. 
Marsh, H.W., \& Hocevar, D. (1985). Application of confirmatory factor analysis to the study of self-concept: First- and higher-order factor models and their invariance across groups. Psychological Bulletin, 97, 562-582. Doi: https://doi.org/10.1037/0033-2909.97.3.562 Nadal, B. (2015). Las inteligencias múltiples como una estrategia didáctica para atender a la diversidad y aprovechar el potencial de todos los alumnos. Revista nacional e internacional de educación inclusiva, $8(3), 121-136$

Naranjo, M. L. (2009). Motivación: perspectivas teóricas y algunas consideraciones de su importancia en el ámbito educativo. Revista Educación 33(2), 153-170. Doi: https://doi.org/10.15517/revedu. v33i2.510

Olaz, F. (2001). La teoría social cognitiva de la autoeficacia. Contribuciones a la explicación del comportamiento vocacional (Trabajo de grado). Universidad Nacional de Córdoba. Argentina

Olaz, F. (2003). Modelo social cognitivo del desarrollo de carrera. Evaluar, 3(1), 15-34.

Pérez, E. (2001). Construcción de un inventario de autoeficacia para inteligencias múltiples. (Tesis Doctoral). Universidad Macional de Córdova, Argentina

Pérez, E., Beltramino, C., \& Cupani, M. (2003). Inventario de Autoeficacia para Inteligencias Múltiples: Fundamentos teóricos y estudios psicométricos. Evaluar, 3(1), 35-60.

Pérez, L., \& Beltrán, J. (2006). Dos décadas de «inteligencias múltiples»: implicaciones para la psicología de la educación. Papeles del Psicólogo, 27(3), 147-164.

Pérez, E., \& Cupani, M. (2008). Validación del Inventario de Autoeficacia para Inteligencias Múltiples Revisado (IAMI-R). Revista Latinoamericana de Psicología, 40(1), 47-58.

Pérez, E., Cupani, M., \& Ayllón, S. (2005). Predictores de rendimiento académico en la escuela media: habilidades, autoeficacia y rasgos de personalidad. Avaliação Psicológica, 4(1), 1-11.

Pérez, E., \& Medrano, L. (2007). Investigación de Autoeficacia para Inteligencias Múltiples Revisado: Un estudio de validez de criterio. Avances en Medición, 5, 105-114. 
Pérez, E., Lescano, C., Heredia, D., Zalazar, P., Furlám, L., \& Martínez, M. (2011). Desarrollo y análisis psicométricos de un Inventario de Autoeficacia para Inteligencias Múltiples en Niños Argentinos. Psicoperspectivas, 10 (1), 169-189. Recuperado de: http://www. psicoperspectivas.cl/index.php/psicoperspectivas/article/view/141/169

Piergiovanni, L. F., \& Depaula, P. D. (2018). Self-efficacy and tress coping styles in university students. Ciencias Psicológicas, 12(1), 17 - 23. Doi: https://doi.org/10.22235/cp.v12i1.1591

Raykov, T., \& Marcoulides, G. A. (2011). Introduction to psychometric theory. Nueva York: Routledge.

$\mathrm{R}$ Core Team (2018). R: A language and environment for statistical computing. Viena: R Foundation for Statistical Computing.

Schumacker, R. E., \& Lomax, R. G. (2016). A Beginner's Guide to Structural Equation Modeling (4th Edition). New York: Routledge Taylor and Francis Group, LLC.

Schunk, D. H. (2012). Teorías del aprendizaje. Una perspectiva educativa (6 ${ }^{\mathrm{a}}$ Ed.). México: Pearson Educación

Shearer, B. (1995). The MIDAS manual. U.S. Departament of Education: Nacional Institutte on Disability and Rehabilitation Research.

Tabachnick, B., \& Fidell, L. (2001). Using multivariate statistics. Fourth edition. Boston: Allyn and Bacon.

Zalazar, M., Aparicio, M., Ramírez, C., \& Garrido, S. (2011). Estudios preliminares de adaptación de la Escala de Fuentes de Autoeficacia para Matemáticas. Revista Argentina de Ciencias del Comportamiento, 3(2), 1-6. 